\title{
Effect of Surfactants on the Oscillatory Behaviour of Metal-ion Catalyzed Pyrogallol based Belousov- Zhabotinsky Reaction
}

\author{
NISAR AHMAD FARHAD, G. M. PEERZADA* and NADEEM BASHIR GANAIE \\ Department of Chemistry, University of Kashmir, Srinagar-190006 (J\&K), India \\ peerzada_gmp@yahoo.co.in
}

Received 24 March 2014 / Accepted 21 April 2014

\begin{abstract}
The present investigation pertains to the effect of surfactants on the oscillatory behavior of the catalyzed Belousov-Zhabotinsky (BZ) reaction having pyrogallol as organic substrate in a continuous stirred tank reactor (CSTR) at $30 \pm 0.1{ }^{\circ} \mathrm{C}$. The surfactants used in this study are sodium dodecyl sulphate (SDS) as the anionic surfactant, cetyl trimethylammonium bromide (CTAB) as the cationic surfactant and triton $\mathrm{x}-100$ (TX-100) as the neutral surfactant. The results revealed that the addition of the surfactants above their critical micelle concentration $(\mathrm{cmc})$ influenced the oscillatory parameters with induction period showing a definite variation with the increasing concentration of aforesaid surfactants. It is noteworthy to mention that the ability of micelles to selectively compartmentalize ions and molecules may account for their effects on the induction period, amplitude and time period of the BZ system under investigation.
\end{abstract}

Keywords: Bromate oscillator, Pyrogallol, Micelles, Oscillations

\section{Introduction}

The oscillatory chemical reactions which are highly complex and far from equilibrium dynamic systems, have been the focus of much research in the area of theoretical and experimental chemical kinetics in recent years ${ }^{1-3}$. These oscillatory chemical reactions have been observed in both homogeneous and heterogeneous systems. The majority of the processes that occur in the biological systems are characterized by very complex mechanisms of oscillatory chemical reactions. In order to get out of the difficulties present in studying the complex dynamics in biological systems, it is possible to use model systems that though exhibit nonlinear phenomena but is still simpler to study. Among the model systems that have attracted the attention of researchers, the BZ reaction is one of the most extensively studied one, paving way to gain insight into the mechanism of various periodic phenomena taking place in naure ${ }^{4-5}$.

This classical bromate-driven BZ reaction involves the bromination and oxidation of the organic substrate and its derivatives by acidic bromate, catalysed by one electron redox couple mostly like $\mathrm{Ce}^{3+} / \mathrm{Ce}^{4+}, \mathrm{Mn}^{2+} / \mathrm{Mn}^{3+}$ and ferroin/ferrin ${ }^{6}$. The detailed mechanism 
proposed by Field, Koros and Noyes (FKN) have been successful in interpreting essentially all observed behaviours of the BZ reaction and the various bromate oscillators derived from it $^{6}$. Basically, the FKN mechanism contains an inorganic part mainly involving reactions of oxybromine species among themselves and with the metal ion catalyst and an organic part involving reactions of organic substrate and its derivatives with Ce(IV) and oxybromine species. The principal features of the FKN mechanism are strongly supported by modeling computations of Edelson et al., ${ }^{7,8}$ and Field et al. ${ }^{9}$

An promising area of recent experimental focus in the nonlinear oscillating chemical reaction is control over its dynamics and this control can be achieved internally by performing reactions in the presence of non-reacting chemical species such as micelles ${ }^{10-16}$ which are known to affect chemical equilibria and reactivity by selectively sequestering the reagent substrates by means of electrostatic and hydrophobic interactions ${ }^{17,18}$. Investigating the influence of micelle forming surfactants on the BZ oscillatory reactions has received growing attention not only for the attempt to elucidate their complex mechanism ${ }^{19,20}$ but also for their relevance to the periodic phenomena in biochemical systems ${ }^{21,22}$. In fact, one of the most important properties of micellar solutions is their ability to selectively interact with the species present in the medium depending on their chemical properties and solubilization sites viz., the hydrophilic shell, the palisade layer and the hydrophobic core. In this way the nature of the solubilized species dictate the locus of solubilization and thus hydrophilic species prefer to stay in the hydrophilic shell, amphiphillic molecules stay preferentially with the polar part in contact with micellar surface and the hydrophobic portion is directed towards the core while as the hydrophobic one is much more localized in the core ${ }^{23}$. This way, by studying the behavior of the oscillating BZ systems in presence of micelles, valuable information to elucidate the reaction mechanism and the nonlinear phenomena can be obtained.

Keeping this into consideration and with an aim to obtain insight into the type of interactions involved in the binding of the reactants to the micellar aggregates and the role played by micelles of various surfactant systems by influencing the dynamical behavior of the $\mathrm{BZ}$ reaction, we have undertaken the present experimental work in order to study the effect of ionic/polar head group of micelles of various surfactants on the kinetics of $\mathrm{Mn}^{2+}$ catalyzed pyrogallol based BZ system through CSTR.

\section{Experimental}

All reagents used were either analytical grade chemicals or else of high purity. The reagents used were pyrogallol 99\% (SRL, AR), potassium bromate 99.6\% (Merck, AR), manganese(II) sulphate monohydrate 98\% (B.D.H, LR) and sulphuric acid 98\% (Merck, LR). The different surfactants i.e., SDS 99\% (Fluka, AR), CTAB 99\% (Sigma Aldrich, AR) and TX-100 98\% (Himedia, LR) were used as received. The solutions of the desired reagents were prepared in $1.5 \mathrm{~mol} \mathrm{~L}^{-1}$ sulphuric acid as aqueous acid medium.

\section{Methods}

The instrumental set up used to monitor the oscillatory behaviour of the aforesaid BZ system consists of a cylindrical reaction vessel made up of glass kept in a high precision water bath (Advantec TBS 181SB) with a compatible magnetic stirrer (Advantec SRS 311AA) to reach the desired temperature and stirring conditions. A magnetic stir bar $(8 \mathrm{~mm}$, Cole Parmer04765-55) was used to achieve uniform stirring at $600 \mathrm{rpm}$. The reactants were added to the reaction vessel and the products were lead away from it by using a Model C.P. 78001-02 system were used to deliver the reactants and the two channels were used to remove the four 
channel peristaltic pump at a specific flow rate of $0.7 \mathrm{~mL} / \mathrm{min}$. Two channels in the waste to keep the total volume of reaction mixture in the reaction vessel constant at $20 \mathrm{~mL}$. The oscillations were monitored by a solid state bromide ion selective electrode (ELIT 2014) and a double junction $\mathrm{Ag} / \mathrm{AgCl}$ reference electrode (ELIT 002N $\mathrm{KNO}_{3}$ 59421), which were directly connected to a four channel ion analyser (ELIT 9804).

The cmc values in these reaction conditions were determined from surface tension $(\gamma)$ versus log of surfactant concentration. The surface tension measurements were made with the Kruss 9 Tensiometer by the platinum ring detachment method and the temperature was maintained at $30 \pm 0.1{ }^{\circ} \mathrm{C}$ by circulating water from a Hake $\mathrm{GH}$ thermostat.

\section{Results and Discussion}

The organized surfactant assemblies are well known to effect chemical equilibria and reactivity by selectively sequestering the reagent substrates by means of electrostatic and hydrophobic interactions. The chemical oscillating reactions involve ionic reactants and intermediates, which are expected to interact with oppositively charged ionic micelles and leads to a consequent changes in both the reaction kinetics and the characteristic oscillatory properties. Figure 1 shows the potentiometric time series of the pyrogallol based BZ system in presence of varying concentrations of SDS. A sudden initial rise and fall in potential/ or bromide ion concentration (off scale in Figure 1) were present in all the oscillatory profiles when the different concentrations of SDS are added to the present BZ reaction mixture.
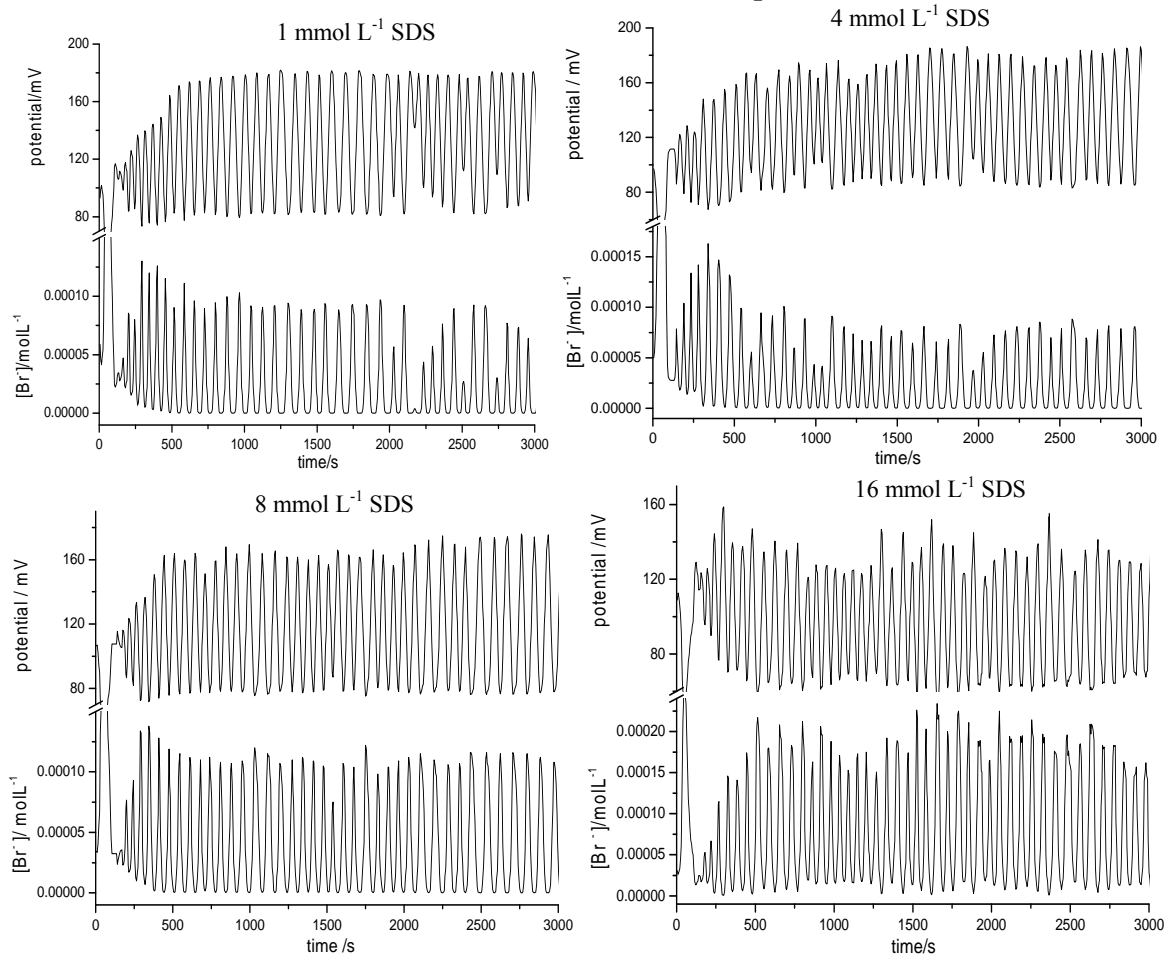

Figure 1. Plots showing the effect of varying concentrations of SDS on the oscillatory behaviour of $\mathrm{BZ}$ system containing [Pyrogallol] $=0.009 \mathrm{~mol} \mathrm{~L}^{-1},\left[\mathrm{Mn}^{2+}\right]=0.005 \mathrm{~mol} \mathrm{~L}^{-1}$, $\left[\mathrm{BrO}_{3}{ }^{-}\right]=0.09 \mathrm{~mol} \mathrm{~L}^{-1},\left[\mathrm{H}_{2} \mathrm{SO}_{4}\right]=1.5 \mathrm{~mol} \mathrm{~L}^{-1}$ at $30 \pm 0.1^{\circ} \mathrm{C}$ in a CSTR 
This sudden initial rise and fall of potential/ or bromide ion concentration is due to the initial liberation of bromine ${ }^{24,25}$. The choice of SDS has been mainly dictated by two of its characteristics: i) it does not react with the components of the BZ systems and ii) its capability to change the aggregation form only as a function of its concentration ${ }^{26}$. The cmc values of different surfactants obtained in the present reaction conditions were $\mathrm{SDS}=0.0288 \mathrm{mmol} \mathrm{L}^{-1}, \mathrm{CTAB}=$ $0.0191 \mathrm{mmol} \mathrm{L}^{-1}$ and TX-100 $=0.051 \mathrm{mmol} \mathrm{L}^{-1}$. The lower cmc value can be attributed to the very high value of ionic strength which influences the micellization process. Further, a definite variation of induction period (IP) is observed which depends upon the nature and concentration of various surfactants added. As is evident from the data in Table 1, for SDS the IP increases, in case of CTAB the IP decreases up to $2 \mathrm{mmol} \mathrm{L}^{-1}$ concentration after which an abrupt increase in IP is observed, whereas in TX-100 a decrease in the IP is observed.

Table 1. Induction Period(s) of the BZ reaction containing [Pyrogallol] $=0.009 \mathrm{~mol} \mathrm{~L}^{-1}$, $\left[\mathrm{Mn}^{2+}\right]=0.005 \mathrm{~mol} \mathrm{~L}^{-1},\left[\mathrm{BrO}_{3}^{-}\right]=0.09 \mathrm{~mol} \mathrm{~L}^{-1},\left[\mathrm{H}_{2} \mathrm{SO}_{4}\right]=1.5 \mathrm{~mol} \mathrm{~L}^{-1}$ in presence of various concentrations of SDS, CTAB and TX-100 at $30 \pm 0.1{ }^{\circ} \mathrm{C}$ in a CSTR

\begin{tabular}{cccccc}
\hline \multicolumn{2}{c}{ SDS } & \multicolumn{2}{c}{ CTAB } & \multicolumn{2}{c}{ TX-100 } \\
\hline \multicolumn{2}{c}{ Concentration, } \\
mmol L
\end{tabular}

The induction period of the main BZ system is $155 \mathrm{~s}$

The variation of the IP for the BZ reaction in presence of different micellar aggregates can be explained by taking into account their influence on some key steps of the FKN mechanism. According to this mechanism, the IP is observed due to the accumulation of crucial concentration of the organic brominated species prior to the commencement of oscillations. The formation of the bromosubstrate takes place through two processes as per the FKN model as,

$$
\begin{aligned}
& \mathrm{BrO}_{3}^{-}+5 \mathrm{Br}^{-}+6 \mathrm{H}^{+} \rightarrow 3 \mathrm{Br}_{2}+3 \mathrm{H}_{2} \mathrm{O} \\
& 3 \mathrm{Br}_{2}+3 \text { Substrate } \rightarrow 3 \text { Bromosubstrate }+3 \mathrm{Br}^{-}+3 \mathrm{H}^{+}
\end{aligned}
$$

Surfactants being amphiphilic may interact with different BZ species either through tail or head. The hydrophobic tail in case of CTAB is a 16 carbon chain while as SDS contains only a 12 carbon chain. The TX-100 has a polar head group with a long tail containing ether linkages ending in an aromatic group. Thus the order of hydrophobicity is expected to be CTAB $>$ SDS $>$ TX-100.

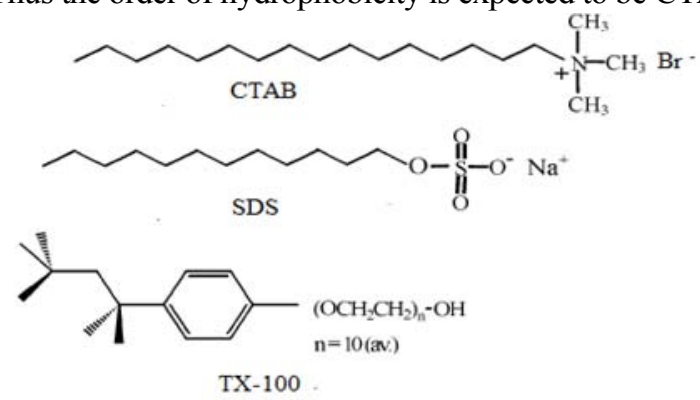

There is a selective interaction between the aggregated system and some crucial species as a result of different solubilization sites, namely the hydrophilic shell, the palisade layer and the hydrophobic core provided by micelles. In case of the SDS, the IP first shows an increase with 
increase in the concentration of the surfactant. The increase in the IP may be due to the repulsive interactions between the negatively charged micellar surface of the SDS and the various negatively charged hydrophilic species $\left(\mathrm{BrO}_{3}{ }^{-}\right.$and $\left.\mathrm{Br}^{-}\right)$and thus the reaction between $\mathrm{BrO}_{3}{ }^{-}$and $\mathrm{Br}^{-}$would preferentially occur in the bulk aqueous phase. Moreover, the replacement of $\mathrm{Na}^{+}$ions on the surface of SDS micelles by $\mathrm{H}^{+}$would decrease the $\mathrm{H}^{+}$ion concentration in the bulk aqueous phase, resulting in an overall decrease in the rate of reaction (1) and consequently the rate of formation of bromosubstrate decreases as is evident from reaction (2). Another reason of increasing the IP may be due to the solubilization of bromine, the most nonpolar species in the BZ system that solubilize in the hydrophobic core of the micelle. The bromine solubilization must result in decrease in the concentration of the bromide ions (i.e., the key inhibiting species in the oscillatory system) in the aqueous phase through the reactions:

$$
\begin{aligned}
2 \mathrm{HBrO}_{2} & \rightarrow \mathrm{BrO}_{3}^{-}+\mathrm{HOBr}+\mathrm{H}^{+} \\
\mathrm{HOBr}+\mathrm{H}^{+}+\mathrm{Br}^{-} & \rightarrow \mathrm{Br}_{2}+\mathrm{H}_{2} \mathrm{O}
\end{aligned}
$$

Further, the evidence of the solubilization of bromine species into aggregated systems has been previously reported for the other reactions ${ }^{27}$.

In presence of cationic $\mathrm{CTAB}$, the rate of reaction (1) is increased due to the localization of negatively charged $\mathrm{BrO}_{3}{ }^{-}$and $\mathrm{Br}^{-}$ions in the palisade layer of the micelles, thereby increasing the rate of reaction (1) with a consequent increase in the rate of reaction (2). This explains the decrease in IP with increasing concentration of surfactant up to $2 \mathrm{mmol} \mathrm{L}^{-1}$ after which an abrupt increase in the IP is observed, which may be both due to the solubilization of bromine and the major involvement of radicals in the BZ mechanism as CTAB with N centre can support such mechanism. Moreover, the polymerization reactions which are driven by BZ systems are well known to involve radical mechanisms ${ }^{28,29}$. In presence of TX-100 micelles, the IP decreases which may be again due to stabilization of negatively charged $\mathrm{BrO}_{3}{ }^{-}$and $\mathrm{Br}^{-}$ ions in the palisade layer of the micelles, thereby increasing the rate of reaction (1). Therefore, the changes in IP with concentration of different surfactant systems can be justified through the electrostatic interaction as well as solubilization tendencies of surfactants towards some important species prevalent in BZ mixture as per the FKN mechanism.

The effect of the different micellar systems on the time period and amplitude was investigated by following the BZ reaction in presence of SDS, CTAB and TX-100 above their $\mathrm{cmc}$ as shown in Figure 2. Tables 2 and 3 show the evolution of time periods and amplitudes in each of these $\mathrm{BZ}$ systems respectively. For the main $\mathrm{BZ}$ reaction, the time period is found to increase (53-90 s) first and then decrease (90-84 s) except for the last oscillations where the time period remains almost constant. However the amplitude shows a continuous increase (52-74 mV) up to $1995 \mathrm{~s}$ after which it remains almost constant. In presence of $16 \mathrm{mM}$ SDS, the time period first increases (38 to $71 \mathrm{~s}$ ), showing a decrease between the time intervals of 1480-1995s after which time period again increased (56-71s). There is also initially a pronounced increase in amplitude (32-76) up to 480 s and then shows a decrease up to $1235 \mathrm{~s}$ after which it remains constant for the last oscillations. In presence of $4 \mathrm{mmol} \mathrm{L}^{-1} \mathrm{CTAB}$, the time period reduces from 88 to $73 \mathrm{~s}$ and then increases from 73 to $87 \mathrm{~s}$ while as the amplitude shows a continuous increase during the course of the reaction. But the most remarkable change is observed when the CTAB was added after the commencement of oscillations showing an increase in bromide ion concentration from $2.55 \times 10^{-4}$ to $2.10 \times 10^{-3} \mathrm{~mol} \mathrm{~L}^{-1}$ with quenching of oscillatory behaviour for about $570 \mathrm{~s}$ after which oscillations with small amplitude were observed as shown in Figure 3. With $1.6 \mathrm{mmol} \mathrm{L} \mathrm{L}^{-1} \mathrm{TX}-100$, the time period shows a continuous increase from 52 to $92 \mathrm{~s}$ and there was significant increase in the amplitude $(101 \mathrm{mV})$ as compared to the main BZ system (80 $\mathrm{mV}$ ) except for the last oscillations where it shows a decrease $(100-91 \mathrm{mV})$. 

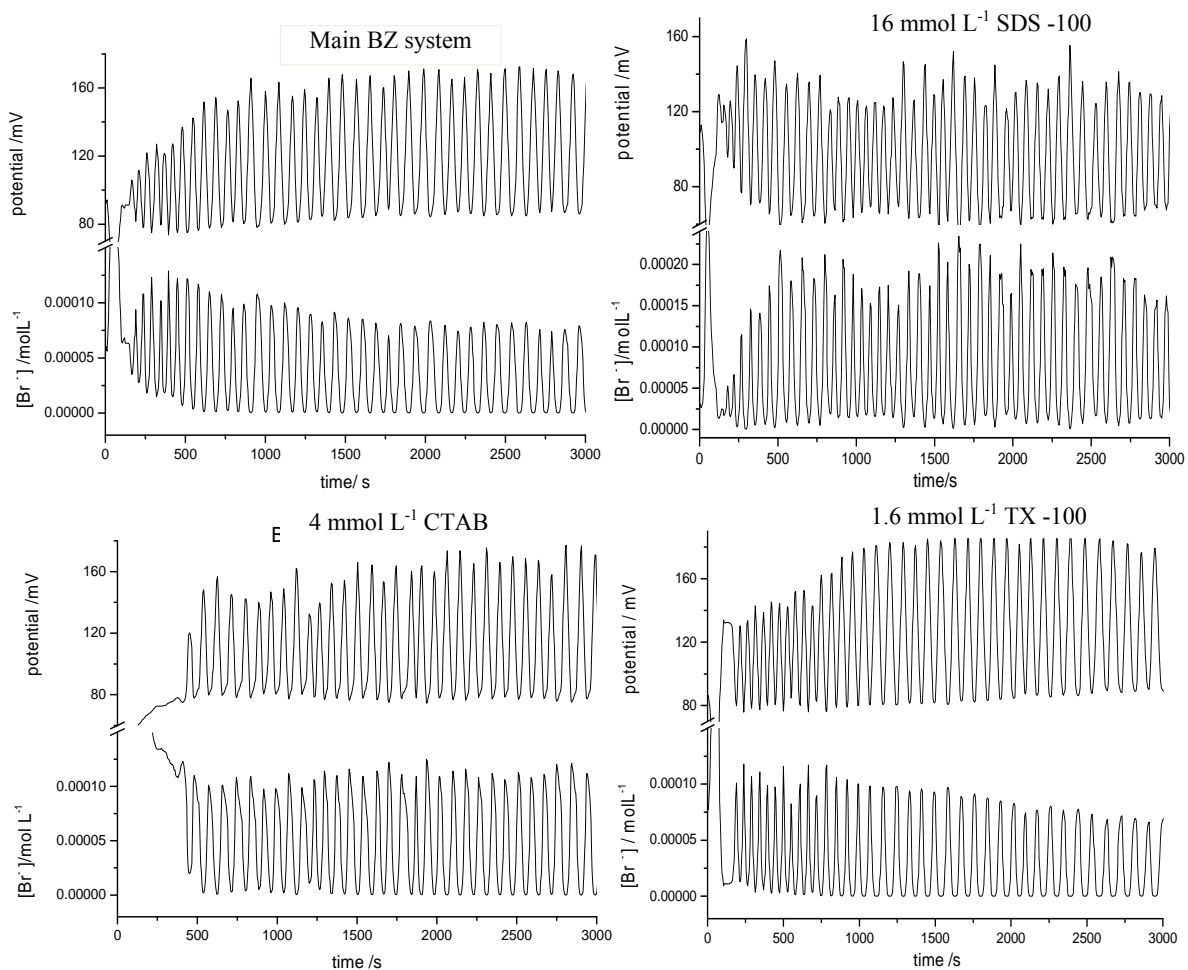

Figure 2. Comparative plots showing the effect of SDS, CTAB and TX-100 on the oscillatory behaviour of $\mathrm{BZ}$ system containing [Pyrogallol] $=0.009 \mathrm{~mol} \mathrm{~L}^{-1},\left[\mathrm{Mn}^{2+}\right]=0.005$ $\mathrm{mol} \mathrm{L}{ }^{-1},\left[\mathrm{BrO}_{3}{ }^{-}\right]=0.09 \mathrm{~mol} \mathrm{~L}^{-1},\left[\mathrm{H}_{2} \mathrm{SO}_{4}\right]=1.5 \mathrm{~mol} \mathrm{~L}^{-1}$ at $30 \pm 0.1^{\circ} \mathrm{C}$ in a CSTR

Table 2. Effect of surfactants on time period of the pyrogallol based BZ reaction system (All the additive concentrations of surfactants were above CMC)

\begin{tabular}{|c|c|c|c|c|c|c|c|}
\hline \multirow{3}{*}{ 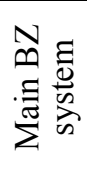 } & Reaction time, $\mathrm{s}$ & $165-485$ & $485-1000$ & $1000-1480$ & $1480-1995$ & $1995-2500$ & $2500-3000$ \\
\hline & $\begin{array}{l}\text { Oscillation time } \\
\text { period (average), s }\end{array}$ & 53 & 73 & 80 & 90 & 84 & 85 \\
\hline & $\begin{array}{l}\text { Oscillation time } \\
\text { period (range), s }\end{array}$ & $50-60$ & $65-80$ & $75-85$ & $85-100$ & $90-80$ & $80-90$ \\
\hline \multirow{3}{*}{ 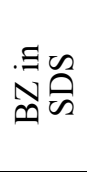 } & Reaction time, $\mathrm{s}$ & $125-240$ & $240-480$ & $480-955$ & $955-1235$ & $1235-2020$ & $2020-2950$ \\
\hline & $\begin{array}{l}\text { Oscillation time } \\
\text { period (average), } \mathrm{s}\end{array}$ & 38 & 60 & 71 & 56 & 66 & 71 \\
\hline & $\begin{array}{l}\text { Oscillation time } \\
\text { period (range), s }\end{array}$ & $35-45$ & $55-65$ & $70-75$ & $60-55$ & $60-75$ & $65-85$ \\
\hline \multirow{3}{*}{ 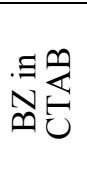 } & Reaction time, $s$ & $450-805$ & $805-1205$ & $1205-1420$ & $1420-1745$ & $1745-2475$ & $2475-2905$ \\
\hline & $\begin{array}{l}\text { Oscillation time } \\
\text { period (average), } \mathrm{s}\end{array}$ & 88 & 80 & 73 & 81 & 83 & 87 \\
\hline & $\begin{array}{l}\text { Oscillation time } \\
\text { period (range), } s\end{array}$ & $85-90$ & $75-85$ & $80-65$ & $75-90$ & $80-95$ & $80-95$ \\
\hline \multirow{3}{*}{ 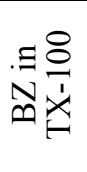 } & Reaction time, $\mathrm{s}$ & $215-530$ & $530-1035$ & $1035-1540$ & $1540-2055$ & $2055-2585$ & $2585-2950$ \\
\hline & $\begin{array}{l}\text { Oscillation time } \\
\text { period (average), } s\end{array}$ & 52 & 63 & 84 & 86 & 88 & 92 \\
\hline & $\begin{array}{l}\text { Oscillation time } \\
\text { period (range), s }\end{array}$ & $50-55$ & $60-70$ & $80-90$ & $80-90$ & $75-95$ & $90-95$ \\
\hline
\end{tabular}


Table 3. Effect of surfactants on amplitude of pyrogallol based BZ reaction system (All the additive concentrations of surfactants were above CMC)

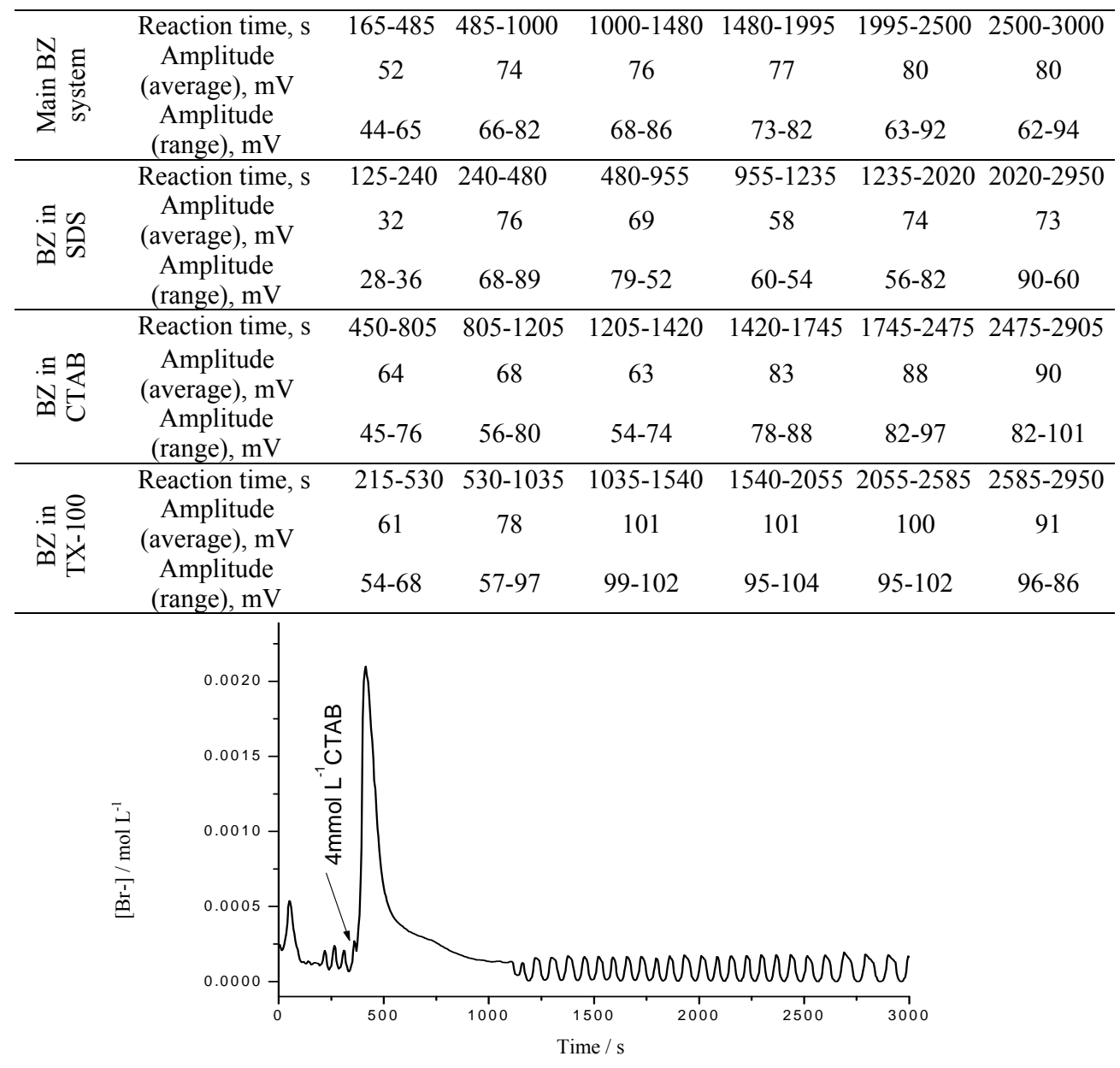

Figure 3. Plot showing the effect of $\mathrm{CTAB}$ added after the commencement of oscillations on the $\mathrm{BZ}$ system containing [Pyrogallol] $=0.009 \mathrm{~mol} \mathrm{~L}^{-1},\left[\mathrm{Mn}^{2+}\right]=0.005 \mathrm{~mol} \mathrm{~L}^{-1},\left[\mathrm{BrO}_{3}^{-}\right]=0.09$ $\mathrm{mol} \mathrm{L}-1,\left[\mathrm{H}_{2} \mathrm{SO}_{4}\right]=1.5 \mathrm{~mol} \mathrm{~L}^{-1}$ at $30 \pm 0.1{ }^{\circ} \mathrm{C}$ in a CSTR. (Arrow indicate the point of injection)

The results of such a study (Figure 2 ) show that it is possible to change the course of the $\mathrm{BZ}$ reaction in presence of non -reacting aggregates such as micelles. This is expected, as surfactants with varying charges and micelle environment would have differing effects on the various reactive species, hence altering the kinetics of the multiple nonlinearly coupled steps in the BZ reaction. Further, the binding constants of various BZ species to micelle will depend on the charge/ or nature of the surfactant, nature of the ionic/ neutral species and the ionic strength of the medium. Also, the dissociation rates of micelle into individual monomers would be different, depending on the nature of the surfactant and ionic strength of the medium ${ }^{30}$. Moreover, the compartmentalization of various BZ species in micelle have been shown to markedly affect the oscillatory characteristics of the BZ reaction. Also, the effect of micelles on diffusion coefficient of BZ species and density of the medium would 
change the transport properties of various ionic/ neutral species generated during the course of $\mathrm{BZ}$ reaction ${ }^{31-33}$. A more captivating possibility is that micelles of different surfactants are well known to affect the bromide ion regeneration step i.e., process $\mathrm{C}$, in the $\mathrm{FKN}$ mechanism.

$\mathrm{M}($ oxd $)+$ Substrate + Bromosubstrate $\rightarrow \mathrm{M}($ red $)+\mathrm{fBr}^{-}+$organic oxidation products

$$
\text { e.g. } \mathrm{HCOOH}, \mathrm{CO}_{2} \text {, etc., }
$$

Edelson and Thomas concluded from their sensitivity analysis of the BZ reaction that the oscillation time period is most sensitive to this step $^{34}$. All these factors would affect the oscillatory characteristics and change the course of the present BZ reaction. Thus, various surfactants would have different effects of varying magnitudes as supported by our findings in Figure 2. It is therefore concluded that micelles of various surfactants control the oscillatory parameters like induction period, time period and amplitude of the present BZ system and the nature of surfactant head group region, including the charge and presence of $\mathrm{N}$ centers along with other hydrodynamic properties play an important role in affecting the concentration of various species and the rates of different steps in a well stirred BZ reaction.

\section{Conclusion}

The pyrogallol based BZ system responds in a peculiar way to the addition of increasing concentrations of different surfactants in a CSTR. The values of oscillatory parameters i.e., induction period, amplitude and time period, depend on concentration of the surfactant used. The observed effects are due to the ability of micelles to selectively sequester some key reacting species in a BZ reaction. With increasing concentration of SDS, a continuous increase in IP is observed, in case of CTAB, the IP first decreases and then shows an abrupt increase, whereas in TX-100 there is a continuous decrease in the IP. Moreover, the nature of ionic/polar headgroup and other hydrodynamic processes of the micellar environment control the overall course of the BZ system.

\section{Acknowledgement}

The authors are highly thankful to UGC, New Delhi for providing financial support in the form of a major research project, owing to which we could make the required facilities available for undertaking the present investigation.

\section{References}

1. Prieto R J, Silva M and Bendito D P, Anal Chem., 1995, 67(4), 729-734; DOI:10.1021/ac00100a007

2. $\quad$ Field R J, J Chem Educ., 1972, 49(4), 308; DOI:10.1021/ed049p308

3. Field R J and Schneider F W, J Chem Educ., 1989, 66(3), 195; DOI:10.1021/ed066p195

4. Scott S K, Oscillations, Waves and Chaos in Chemical Kinetics, Oxford Chemistry Primers 18, Oxford University Press, Oxford, UK, 1994.

5. Field R J and Burger M, Oscillations and Traveling Waves in Chemical Systems, Wiley, New York, 1985.

6. $\quad$ Field R J, Koros E and Noyes R M, J Am Chem Soc., 1972, 94(25), 8649-8664; DOI:10.1021/ja00780a001

7. Edelson D, Field R J and Noyes R M, Int J Chem Kinet., 1975, 7(3), 417-432; DOI:10.1002/kin.550070309

8. Edelson D, Noyes R M and Field R J, Int J Chem Kinet., 1979, 11(2),155-164; DOI:10.1002/kin.550110207 
9. Gyorgyi L, Turany T and Field R J, J Phys Chem., 1990, 94, 7162-7170; DOI: $10.1021 / \mathrm{j} 100381 \mathrm{a} 039$

10. Vanag V K and Hanazaki I, J Phys Chem A, 1997, 101(11), 2147-2152; DOI:10.1021/jp9637183

11. Sciascia L, Lombardo R and Liveri M, J Phys Chem B, 2007, 111(6), 1354-1360; DOI:10.1021/jp067052g

12. Maritato M, Nikles J, Romsted L S and Tramontin M, J Phys Chem., 1985, 89, 1341-1344.

13. Vanag V K and Epstein I R, Phys Rev Lett., 2001, 87, 228301.

14. Paul A, J Phys Chem B, 2005, 109(19), 9639-9644; DOI:10.1021/jp044519j

15. Najar M H, Dar A A and Rather G M, Int J Chem Kinet., 2010, 42(11), 659-668; DOI:10.1002/kin.20514

16. Kupitz D, Alonso S, Bar M and Hauser M J B, Phys Rev E., 2011, 84, 056210.

17. Bunton C A and Savelli G, Adv Phys Org Chem., 1986, 22, 213-309; DOI:10.1016/S0065-3160(08)60169-0

18. Burgess J and Pelizzetti E, Gazz Chim Ital., 1988, 118, 803.

19. Gyorgyi L, Turanyi T and Field R J, J Phy Chem., 1990, 94(18), 7162-7170; DOI:10.1021/j100381a039

20. Benini O, Cervellati $\mathrm{R}$ and Fetto P, Int J Chem Kinet., 1998, 30(4), 291-300; DOI:10.1002/(SICI)1097-4601(1998)30:4<291::AID-KIN8>3.0.CO;2-N

21. Olson D L and Scheeline A, Anal Chim Acta., 1993, 283(2), 703-717; DOI:10.1016/0003-2670(93)85285-R

22. Hung Y F, Schreiber I and Ross J, J Phys Chem., 1995, 99, 1980.

23. Fendler J, Membrane Mimetic Chemistry; John Wiley \& Sons Inc, New York, 1983.

24. Maritato M, Nikles J, Romsted L S and Tramontin M, J Phys Chem., 1985, 89, 1341.

25. Israelachvili J N, Intermolecular and Surfaces Forces, $2^{\text {nd }}$ Ed, Academic Press, New York, 1995.

26. Quina F H, Nassar P M, Bonilha J B S and Bales B L, J Phys Chem., 1995, 99, 17028-17031.

27. (a) Lennox R B and McClelland R A, J Am Chem Soc., 1986, 108(13), 3771-3781; DOI:10.1021/ja00273a035 (b) Cerichelli G, Grande C, Luchetti L, Mancini G and Bunton C A, J Org Chem., 1987, 52(23), 5167-5171; DOI:10.1021/jo00232a020

28. Washington R P, West W W, Misra G P and Pojman J A, J Am Chem Soc., 1999, 121(32), 7373-7380; DOI:10.1021/ja990743o

29. Chowdhury D, Paul A and Chattopadhyay A, J Colloid Interface Sci., 2003, 265(1), 70-76; DOI:10.1016/S0021-9797(03)00120-6

30. Adamson A W and Gast A P, Physical Chemistry of Surfaces, $6^{\text {th }}$ Ed., John Wiley \& Sons, New York, 1997.

31. Vanag V K and Epstein I R, Phys Rev Lett., 2001, 87, 228301.

32. Rustici M, Lombardo R, Mangone M, Sbriziolo C, Zambrano V and Liveri M T, Faraday Discuss., 2002, 120, 39-51; DOI:10.1039/B103532K

33. Yoshimoto M, Shirahama H, Kurosawa S and Naito M, J Chem Phys., 2004, 120, 7067.

34. Edelson D and Thomas V M, J Phys Chem., 1981, 85(11), 1555-1558; DOI:10.1021/j150611a019 